\title{
Sisyphus cooling and amplification by a superconducting qubit
}

\author{
M. GRAJCAR ${ }^{1,2 *}$, S. H. W. VAN DER PLOEG ${ }^{1}$, A. IZMALKOV ${ }^{1}$, E. L'I'ICHEV' ${ }^{1}$, H.-G. MEYER ${ }^{1}$, A. FEDOROV ${ }^{3}$, \\ A. SHNIRMAN ${ }^{4}$ AND GERD SCHÖN 5 \\ ${ }^{1}$ Institute of Photonic Technology, PO Box 100239, D-07702 Jena, Germany \\ ${ }^{2}$ Department of Experimental Physics, Comenius University, SK-84248 Bratislava, Slovakia \\ ${ }^{3}$ Kavli Institute of Nanoscience, Delft University of Technology, PO Box 5046, 2600 GA, Delft, The Netherlands \\ ${ }^{4}$ Institut für Theoretische Physik, Universität Innsbruck, A-6020 Innsbruck, Austria \\ ${ }^{5}$ Institut für Theoretische Festkörperphysik and DFG-Center for Functional Nanostructures (CFN), Universität Karlsruhe, D-76128 Karlsruhe, Germany \\ *e-mail: grajcar@fmph.uniba.sk
}

Laser cooling of atoms paved the way for remarkable achievements in quantum optics, including Bose-Einstein condensation and trapping in optical lattices. Recently, superconducting qubits-micrometre-size superconducting circuits-were shown to act as artificial atoms, exhibiting quantum effects such as Rabi oscillations and Ramsey fringes ${ }^{1-3}$. Coupling superconducting circuits to resonators brought them into the realm of quantum electrodynamics ${ }^{4-7}$ and opened up perspectives for using them as micro-coolers or to create a population inversion inducing lasing behaviour ${ }^{8-12}$. Here, we demonstrate so-called Sisyphus cooling ${ }^{13}$ and amplification of an $L C$ resonator, which consists of an inductor $L$ and a capacitor $\mathrm{C}$, by a superconducting qubit, furthering the analogies between optical and circuit quantum electrodynamics. In quantum optics, the motion of the atom is cooled or amplified by a laser driving its electronic degrees of freedom. In our system, the roles of the two degrees of freedom are played by the levels of the resonator and the qubit. Red-detuned high-frequency driving of the qubit produces cooling, because the low-frequency LC circuit carries out work in the forward and backward oscillation cycle, always increasing the energy of the qubit. For blue-detuning, the same mechanism leads to Sisyphus amplification and a precursor of lasing. Parallel to the experimental demonstration, we analyse these processes theoretically, quantitatively confirming our interpretation.

The system considered is shown schematically in the inset of Fig. 1a with a scanning electron micrograph provided in Fig. 1 b. It consists of a three-junction flux qubit ${ }^{14}$ with the two qubit states corresponding to persistent currents of amplitude $I_{\mathrm{p}}$ flowing clockwise and anticlockwise. When operated in the vicinity of the degeneracy point, $f_{x} \equiv \Phi_{x} / \Phi_{0}-1 / 2 \approx 0$, where $\Phi_{x}$ is the magnetic flux applied to the qubit loop and $\Phi_{0}=h / 2 e$ is the flux quantum, the Hamiltonian of the qubit in the basis of the persistent current states reads

$$
H=-\frac{1}{2} \varepsilon\left(f_{x}\right) \sigma_{z}-\frac{1}{2} \Delta \sigma_{x}
$$

Here, $\sigma_{i}$ are Pauli matrices, $\Delta$ is the tunnelling amplitude and $\varepsilon\left(f_{x}\right)=2 \Phi_{0} I_{\mathrm{p}} f_{x}$ is the energy bias. The energy levels of the isolated qubit, separated by $\Delta E\left(f_{x}\right)=\sqrt{\varepsilon^{2}\left(f_{x}\right)+\Delta^{2}}$, are shown in Fig. 1 .
The qubit is driven by a high-frequency field with frequency $\omega_{\mathrm{d}}$ and coupled through a mutual inductance $M$ to a low-frequency tank circuit with frequency much lower than the level spacing of the qubit, $\hbar \omega_{\mathrm{T}} \ll \Delta$. Both the high-frequency driving and the coupling to the tank circuit can be included at this stage through their contributions to the external flux $\Phi_{x}$. The slowly oscillating current in the tank circuit can be treated in an adiabatic approximation, that is, it shifts the bias flux of the qubit by $\Phi_{x}(t)=M\left[I_{\text {d.c. }}+I_{\text {r.f. }}(t)\right]$. To illustrate the principle, we first treat $I_{\text {r.f. }}(t)$ as a classical a.c. current. For the quantitative description provided below, we will quantize this oscillator degree of freedom.

The system mimics the Sisyphus mechanism of damping (cooling) and amplification (tendency towards lasing) known from quantum optics ${ }^{13}$. This mechanism is shown in Fig. 1. Here, we describe the damping (cooling, hence marked in blue) for a situation where the driving is red-detuned, $\hbar \omega_{\mathrm{d}}<\Delta E$; the amplification (marked in red) for blue-detuning can be described in an analogous way. The oscillations of the current in the tank circuit, $I_{\mathrm{r} . \mathrm{f}}(t)$, lead to oscillations of $\varepsilon\left(f_{x}\right)$ around a value determined by the d.c. component, $I_{\text {d.c. }}$. In the first part of the cycle, when the qubit is in the ground state, the current shifts the qubit towards the resonance at $\Delta E=\hbar \omega_{\mathrm{d}}$. The energy of the qubit grows owing to the work done by the inductor-capacitor (LC) circuit. Once the system reaches the vicinity of the resonance point, the qubit can be excited, the energy being provided by the high-frequency driving field. With parameters adjusted such that this happens at the turning point of the oscillating trajectory, in the following the qubit in the excited state is shifted away from the resonance, such that the qubit's energy continues to grow. Again the work has to be provided by the LC circuit. The cycle is completed by a relaxation process that takes the qubit back to the ground state, while the work carried out by the oscillator together with a quantum of energy of the highfrequency driving is released to the environment. The maximum effect is achieved when the driving frequency and relaxation rate are of the same size. If the relaxation is too slow, the state is merely shifted back and forth adiabatically during many periods of oscillations. Note that the complete cycle resembles the ideal Otto-engine thermodynamic cycle ${ }^{15}$. Although the cooling cycle described here differs in some details from the one in atomic 

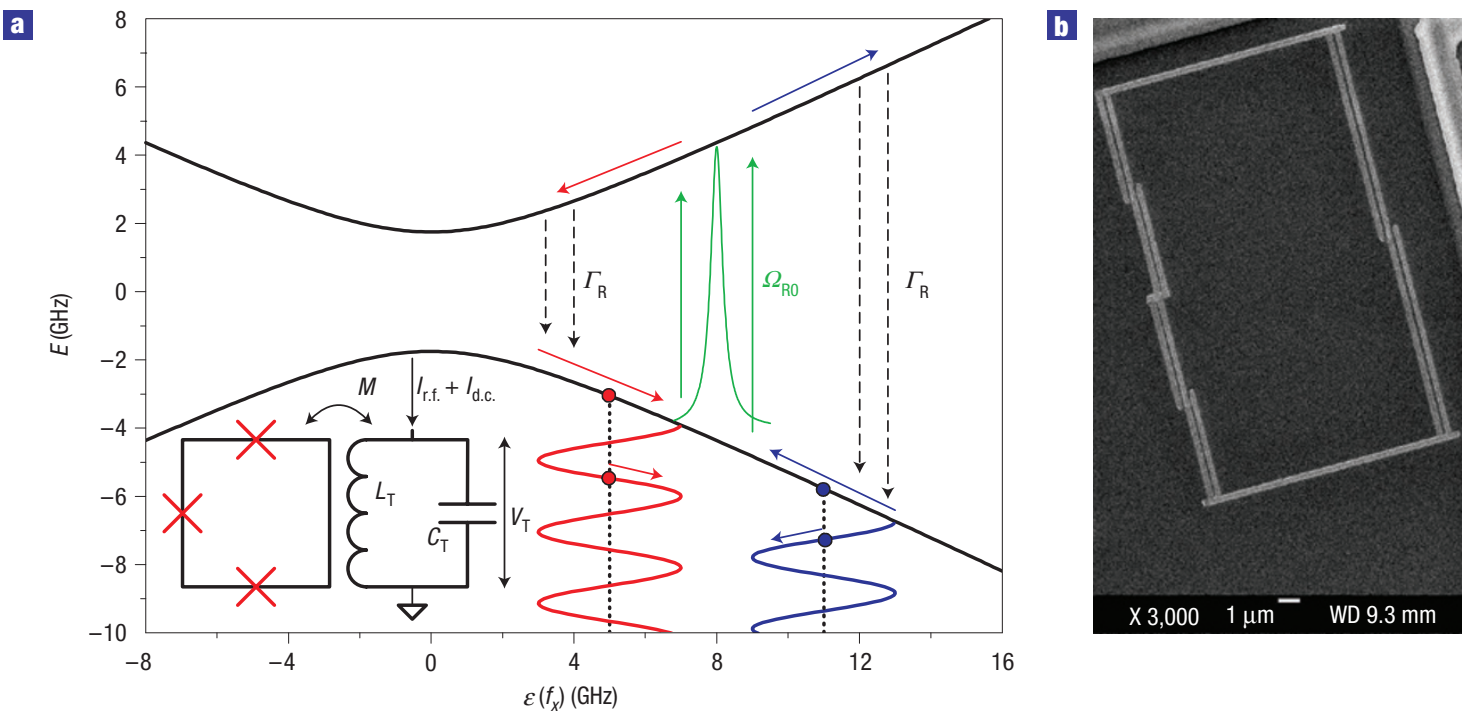

Figure 1 The system and the operation principles. a, The energy levels of the qubit as a function of its energy bias $\varepsilon\left(f_{x}\right)=2 \Phi_{0} l_{p} f_{x}$. The sinusoidal current in the tank coil indicated by wavy lines, with its instantaneous value indicated by the dot, drives the bias of the qubit. The cooling and amplification cycles are marked by blue and red, respectively. The resonant excitation of the qubit due to the high-frequency driving, with amplitude $\Omega_{\mathrm{Ro}}$, is indicated by two green arrows and the Lorentzian depicting the width of this resonance. The frequency of this driving is kept constant, whereas the detuning is controlled by the d.c. bias of the qubit. The relaxation of the qubit is denoted by the black dashed arrows. The inset shows schematically the three-junction qubit coupled to the LC circuit. An onchip microwave antenna (not shown) couples the high-frequency driving to the qubits. $\mathbf{b}$, Scanning electron micrograph of the superconducting flux qubit prepared by the shadow evaporation technique.
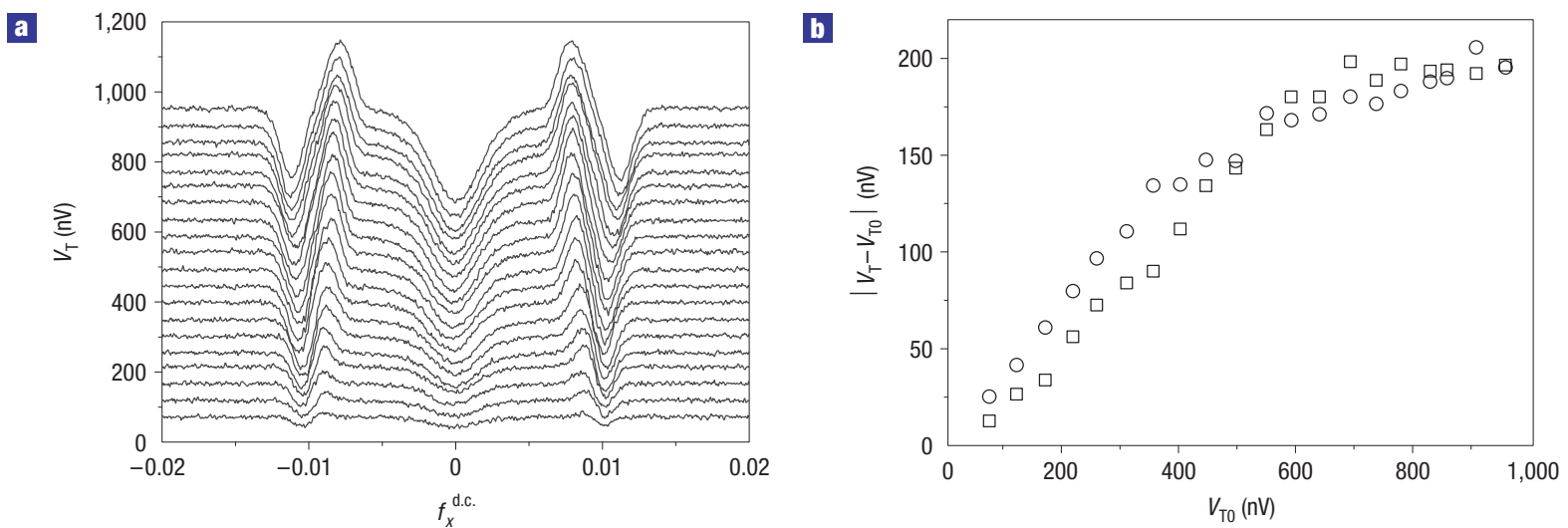

Figure 2 Influence of high-frequency driving of the qubit on the low-frequency response of the tank circuit. a, Amplitude of the radiofrequency tank voltage as a function of the d.c. magnetic bias of the qubit, $f_{x}^{\text {d.c. }}$, for a microwave driving frequency $\omega_{\mathrm{d}} / 2 \pi=14.125 \mathrm{GHz}$ and various amplitudes of the radiofrequency current driving the tank circuit. $\mathbf{b}$, The height of the peaks (squares) and dips (circles) as a function of the voltage amplitude of the unloaded tank circuit $V_{\mathrm{T} 0}$. The voltage $V_{\mathrm{TO}}$ is determined as $V_{\mathrm{T}}$ taken at $f_{x}^{\text {d.c. }}=0.02$ where the effect of the interaction between the tank circuit and the qubit is negligible. The height saturates near $200 \mathrm{nV}$.

systems $^{13}$ where more than two levels are involved and the transition mechanism is different, it demonstrates the basic idea of Sisyphus cooling.

We have carried out two types of measurement. In the first, a response analysis, the LC tank circuit is driven near-resonantly by a low-frequency radiofrequency current, and its response is detected using lock-in techniques. In these measurements, we identify the influence of the high-frequency driven qubit on the effective quality factor and eigenfrequency of the tank circuit. We associate a reduction of the effective quality factor with cooling, whereas the enhancement of the effective quality factor is associated with amplification and tendency towards lasing. Our quantitative analysis enables us to identify parameter regimes where these effect are optimized.

In the second type of measurements, the low-frequency radiofrequency driving is switched off, while the emission of the LC tank circuit is monitored by a spectrum analyser. This analysis probes the influence of the qubit on the effective quality factor, and, most importantly, enables us to determine the energy stored in the tank circuit. Thus, we are able to demonstrate cooling and amplification of the LC oscillator. Note, that the increase of the number of photons in the LC circuit in the amplification regime is not simply heating, because it is accompanied by an increase of the effective quality factor and a 
linewidth narrowing. This property indicates a tendency towards lasing, although the system considered here does not yet reach the lasing instability.

Our results of the first type of experiment are shown in Fig. 2. The dips (peaks) correspond to the Sisyphus damping (amplification) of the tank circuit, that is, to the decrease (increase) of the effective quality factor $Q_{\text {tank }}$. The central dip is due to the shift of the oscillator frequency when the qubit is at its degeneracy point ${ }^{16}$ and is not related to the Sisyphus effect. We observe from Fig. $2 b$ that the extra voltage saturates for large driving amplitudes at approximately $200 \mathrm{nV}$. Using this value and equation (6) (see the Methods section), we observe that indeed the relaxation time is close to the period of oscillations, $\Gamma_{\mathrm{R}} \approx 0.2 \omega_{\mathrm{T}}$ or $\Gamma_{\mathrm{R}} \approx 0.8 \omega_{\mathrm{T}}$ (two solutions are allowed by equation (6)). We also note that the distance between the points of optimal damping and optimal amplification grows with the amplitude of the external low-frequency driving. This confirms our conjecture that the cooling (amplification) is optimized when the oscillating current, $I_{\text {r.f. }}(t)$, brings the qubit into the resonance with the microwave driving at the turning point of oscillations of $I_{\text {r.f. }}(t)$ (Fig. 1a).

The results for the second type of experiment are shown in Fig. 3. The comparison shows that at the bias point corresponding to maximum Sisyphus damping, the resonant line widens in good agreement with the results for the driven LC circuit. At the bias point corresponding to maximum amplification, the line narrows, indicating a tendency towards lasing. We can extract the quality factors in these two regimes, as well as for the bias point far from the resonance where no extra damping occurs. The comparison of the experimental results with equation (5) of the Methods section section shows a quantitative agreement.

Finally, the results shown in Fig. 3 enable us to estimate the efficiency of the cooling or amplification (lasing) in the two regimes. Integrating the power spectra, we observe that in the damping regime the number of photons in the LC circuit decreases by about $8 \%$ compared with the undamped case. A cooling effect of $6 \%-13 \%$ was observed also for other microwave frequencies. One reason why there is only little cooling lies in the fact that our system is optimized towards maximum Sisyphus damping rather than minimum temperature. Indeed, damping is optimized when the resonant point is reached at the turning point of the oscillator's trajectory. In this regime, even a small reduction of the oscillator's amplitude, that is, weak cooling, switches the whole mechanism off. For more efficient cooling, we could imagine a feedback mechanism in which the detuning is slightly reduced when the $10 \%$ cooling is reached. This would bring the system again into the optimal damping regime with extra cooling.

In our experiments, the qubit's temperature is low, $T_{\mathrm{q}}<100 \mathrm{mK}$, but the tank circuit is strongly coupled to the amplifier anchored at the $T_{0} \approx 4.2 \mathrm{~K}$ stage of the refrigerator. The two orders of magnitude mismatch between the energy level separation of the qubit $\Delta / h \geq 3.5 \mathrm{GHz}$ and that of the LC circuit $\omega_{\mathrm{T}} / 2 \pi \approx 20 \mathrm{MHz}$ makes the coupling between them inefficient for heat transfer. Thus, without active cooling, the oscillator would adopt the high temperature of the amplifier, $T_{\text {tank }} \approx T_{0}$. What we have demonstrated experimentally is the reduction of $T_{\text {tank }}$ by the active Sisyphus cooling by approximately $10 \%$ as compared with $T_{0}$. Potentially, a much stronger cooling could be achieved by first optimizing the Sisyphus cooling as discussed above and then, at a second stage, by switching to, for example, the sideband resolved cooling which could produce $T_{\text {tank }} \sim\left(\omega_{\mathrm{T}} / \omega_{\mathrm{d}}\right) T_{\mathrm{q}} \approx 0.1 \mathrm{mK}$ (ref. 17). Furthermore, the initial temperature of the tank circuit could be reduced by decoupling it from the amplifier, for example, by a radiofrequency isolator. (Unfortunately, cryogenic isolators for the $20-100 \mathrm{MHz}$ range are not available commercially.) Finally,

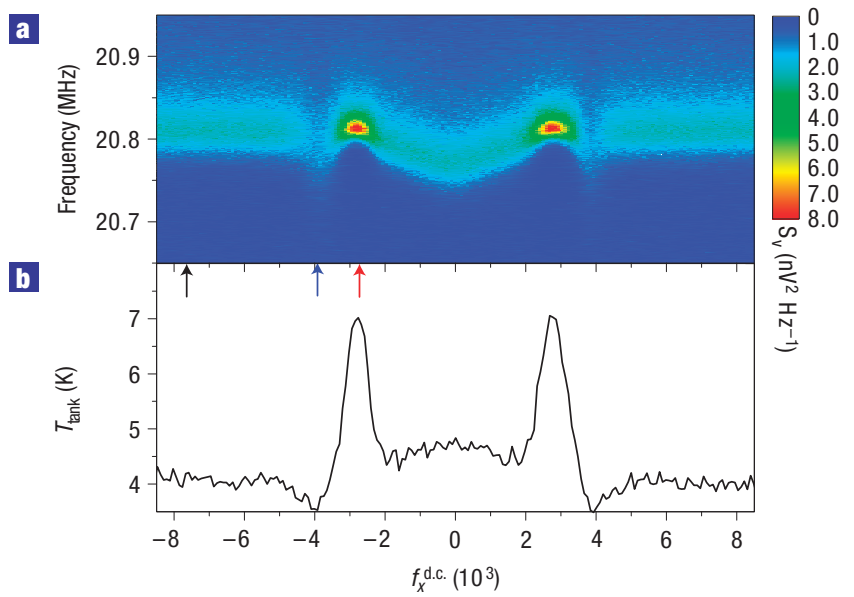

c

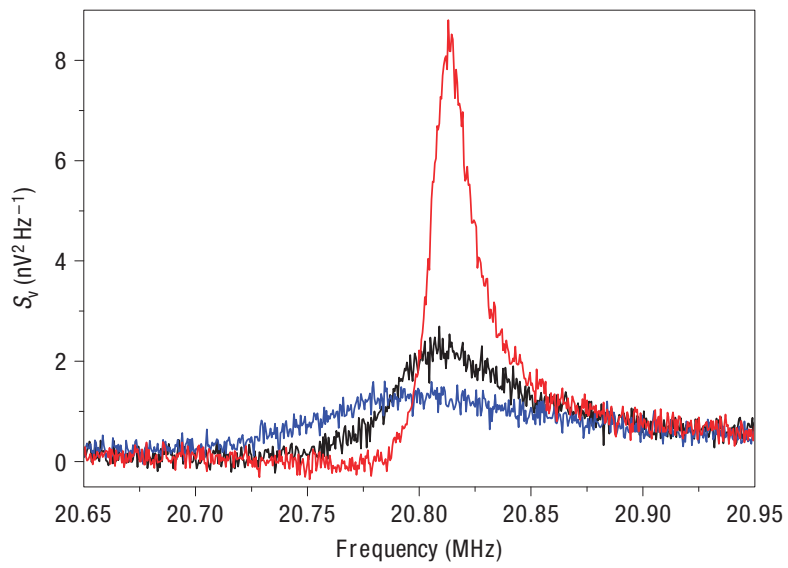

Figure 3 Influence of the driven qubit on the spectral properties of the LC circuit. a, Spectral density of the voltage noise in the LC circuit, $S_{V}(\omega)$, measured when the low-frequency radiofrequency driving is switched off while the high-frequency qubit driving is fixed at $\omega_{\mathrm{d}} / 2 \pi=6 \mathrm{GHz}$. $S_{\mathrm{v}}$ is shown as a function of frequency (vertical axis) and normalized magnetic flux in the qubit $f_{x}^{\text {d.c. }}$ (horizontal axis). $\mathbf{b}$, The integral, $\int S_{\mathrm{V}}(\omega) \mathrm{d} \omega$, evaluated using the data from $\mathbf{a}$, and expressed as the effective temperature of the tank circuit $T_{\text {tank }}$. At the optimal d.c. bias $f_{x}^{\text {d.c. } \text {, }}$ the effective temperature is lower by $8 \%$ than the temperature $T_{0}$ away from the resonance, $\left(T_{0}-T_{\text {tank }}\right) / T_{0}=0.08$. c, Spectral density $S_{\mathrm{V}}(\omega)$ measured at three different values of $f_{x}^{\text {d.c. }}$ corresponding to damping (blue), amplification (red) and away from the resonance (black). These values of $f_{x}^{\text {d.c. }}$ are marked, respectively, by blue, red and black arrows in $\mathbf{a}$.

we expect to gain if, instead of measuring the low-frequency oscillator directly by an amplifier, we infer the information on its state by dispersively measuring the qubit by microwaves in the gigahertz range $^{18-20}$.

To develop the comparison further, we model the Sisyphus damping as an extra effective bath coupled to the oscillator, which we characterize by a quality factor $Q_{\text {Sis }}$ and temperature $T_{\text {Sis }}$. The standard analysis gives the total quality factor and temperature, $Q_{\text {tank }}^{-1}=Q_{0}^{-1}+Q_{\text {Sis }}^{-1}$ and $T_{\text {tank }} Q_{\text {tank }}^{-1}=T_{0} Q_{0}^{-1}+T_{\text {Sis }} Q_{\text {Sis }}^{-1}$, respectively. Here, $T_{0}$ is the temperature of the oscillator without Sisyphus damping, which in our case is determined mostly by the amplifier. Our data show that $Q_{\text {tank }} \sim Q_{0} / 2$. Thus, the coupling strength of the Sisyphus mechanism is comparable to that of the rest of the environment, $Q_{\mathrm{Sis}} \approx Q_{0} \approx 340$. In the present experiment, it seems that $T_{\mathrm{Sis}}$ is comparable to $T_{0}$, consistent with the observation that we have little cooling. 


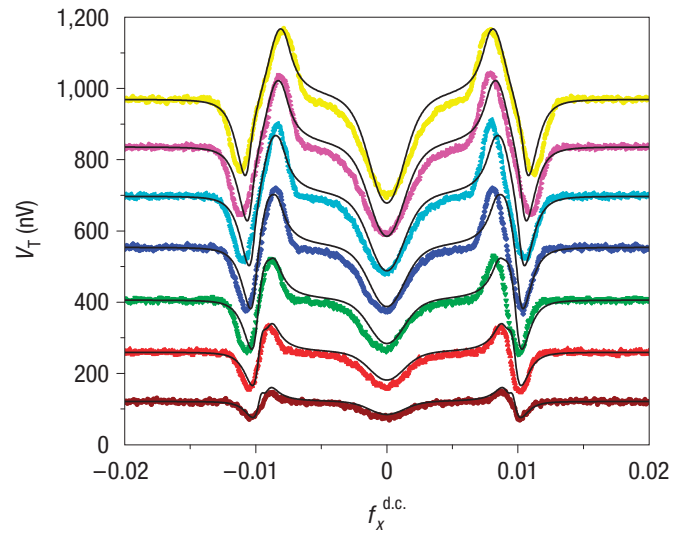

Figure 4 Amplitude of the voltage oscillation for various amplitudes of the radiofrequency driving of the tank circuit. Coloured lines: experimental data. Black lines: numerical solution of equations (3) for $\Omega_{\mathrm{R} 0}=2 \pi \times 0.5 \mathrm{GHz}$, $\Gamma_{\mathrm{R}}=1.0 \times 10^{8} \mathrm{~s}^{-1}, \Gamma_{\varphi}^{*}=5.0 \times 10^{9} \mathrm{~s}^{-1}$. The amplitude of the radiofrequency driving is directly related to the asymptotic value of voltage at $f_{x}^{\text {d.c. }}=0.02$. The central dip is due to the quadratic coupling term in (2) that causes a shift of the oscillator frequency ${ }^{16}$.

In contrast, in the amplification mode of our experiment, the line gets much sharper, which can be formally expressed by choosing $Q_{\text {Sis }}$ negative, $Q_{\text {Sis }} \approx-600$. This means that, although the system did not yet reach the lasing instability, because $\left|Q_{\text {Sis }}\right|>Q_{0}$, it is not far from it. Consistently, the Sisyphus mechanism, acting as an active medium with population inversion, is described by a negative temperature $T_{\text {Sis }}$. Its value is not known, but we can get a lower bound for it from the inequality $T_{\text {tank }} / Q_{\text {tank }}>T_{0} / Q_{0}$. This predicts that the temperature and number of photons should be increased by a factor $\sim 2$. The experiment shows a $36 \%$ increase, which demonstrates the trend, although a quantitative discrepancy remains.

We now outline the theoretical analysis of the problem. Quantizing the oscillations in the tank circuit, we arrive at the Hamiltonian

$$
\begin{aligned}
H= & -\frac{1}{2} \varepsilon\left(f_{x}^{\text {d.c. }}\right) \sigma_{z}-\frac{1}{2} \Delta \sigma_{x}-\hbar \Omega_{\mathrm{R} 0} \cos \left(\omega_{\mathrm{d}} t\right) \sigma_{z} \\
& +\hbar \omega_{\mathrm{T}} a^{\dagger} a+g \sigma_{z}\left(a+a^{\dagger}\right)
\end{aligned}
$$

where the coupling constant is $g=M I_{\mathrm{p}} I_{\mathrm{T}, 0}$ and $I_{\mathrm{T}, 0}=\sqrt{\hbar \omega_{\mathrm{T}} / 2 L_{\mathrm{T}}}$ is the amplitude of the vacuum fluctuation of the current in the LC oscillator. The third term of equation (1) describes the high-frequency driving with amplitude $\Omega_{\mathrm{R} 0}$. After transformations to the eigenbasis of the qubit and some appropriate approximations (described in the Methods section), the Hamiltonian reduces to

$$
\begin{aligned}
H= & -\frac{1}{2} \Delta E \sigma_{z}+\hbar \Omega_{\mathrm{R} 0} \cos \left(\omega_{\mathrm{d}} t\right) \cos \zeta \sigma_{x}+\hbar \omega_{\mathrm{T}} a^{\dagger} a \\
& +g \sin \zeta \sigma_{z}\left(a+a^{\dagger}\right)-\frac{g^{2}}{\Delta E} \cos ^{2} \zeta \sigma_{z}\left(a+a^{\dagger}\right)^{2},
\end{aligned}
$$

with $\Delta E \equiv \sqrt{\epsilon^{2}+\Delta^{2}}$ and $\tan \zeta \equiv \varepsilon / \Delta$. Thus, we obtain the effective Rabi frequency $\Omega_{\mathrm{R} 0} \cos \zeta$ and the effective linear qubit-oscillator coupling $g \sin \zeta$. As we need both of these terms for the Sisyphus cooling, the qubit should be biased near but not exactly at the symmetry point. The second-order term $\propto g^{2}$ is responsible, for example, for the qubit-dependent shift of the oscillator frequency ${ }^{16}$.
We account for the effects of dissipation by the Liouville equation for the density operator of the system, including the two relevant damping terms,

$$
\dot{\rho}=-\frac{i}{\hbar}[H, \rho]+L_{\mathrm{Q}} \rho+L_{\mathrm{R}} \rho .
$$

As far as the qubit is concerned $\left(L_{\mathrm{Q}}\right)$, we consider spontaneous emission with rate $\Gamma_{\mathrm{R}}$ and pure dephasing with rate $\Gamma_{\varphi}^{*}$ (see the Methods section). The damping of the tank circuit $\left(L_{\mathrm{R}}\right)$ is characterized by the friction coefficient $\kappa=\omega_{\mathrm{T}} / Q_{0}$ and effective temperature $T_{0}$ (see the Methods section). We solve the master equation (3) in the quasiclassical limit, that is, for $n \equiv\left\langle a^{\dagger} a\right\rangle \gg 1$, and for various choices of the parameters $\Gamma_{\mathrm{R}}, \Gamma_{\varphi}^{*}$ and $\Omega_{\mathrm{R} 0}$. As the system is harmonically driven, we determine the response of the observables/density matrix at the driving frequency, and, finally, find the amplitude of the driven voltage oscillations across the tank circuit. As shown in Fig. 4, we reproduce well the experimental findings by fitting the system parameters within a reasonable range. Actually, a good fit can be obtained for a relatively wide range of values of the Rabi frequency $\Omega_{\mathrm{R} 0}$ and pure dephasing rate $\Gamma_{\varphi}^{*}$, provided their product is kept roughly constant. On the other hand, the relaxation rate $\Gamma_{\mathrm{R}}$ is determined rather accurately.

The Sisyphus cooling and amplification by a superconducting qubit analysed here can also be applied to nanoelectromechanical systems $^{8,21-25}$. Typical nanomechanical resonators in the frequency range of $10-100 \mathrm{MHz}$ can be coupled to Josephson qubits with coupling strength comparable to that of our system. Owing to typically higher quality factors of nanoelectromechanical systems, more efficient cooling and lasing should be possible.

\section{METHODS}

\section{ESTIMATING THE QUALITY FACTOR}

Here, we provide a qualitative analysis of the first type of measurement, the response analysis. The LC tank circuit is driven by a current source with amplitude $I_{\mathrm{T}}^{\mathrm{d}}$, and the resulting amplitude $V_{\mathrm{T}}$ of the voltage oscillations across the tank circuit is measured. It is

$$
V_{\mathrm{T}}=\omega_{\mathrm{T}} L_{\mathrm{T}} I_{\mathrm{T}}=Q_{\operatorname{tank}} \omega_{\mathrm{T}} L_{\mathrm{T}} I_{\mathrm{T}}^{\mathrm{d}},
$$

where $I_{\mathrm{T}}=\sqrt{2\left\langle I_{\text {r.f. }}^{2}\right\rangle}=Q_{\text {tank }} I_{\mathrm{T}}^{\mathrm{d}}$ is the actual amplitude of the radiofrequency current in the inductance and $Q_{\text {tank }}$ is the effective quality factor of the tank circuit. It is given by the ratio $Q_{\text {tank }}=2 \pi W_{\mathrm{T}} / A$ between the energy stored in the tank, $W_{\mathrm{T}}=L_{\mathrm{T}} I_{\mathrm{T}}^{2} / 2$, and the energy loss $A$ per period. The latter consists of two contributions, $A=A_{\mathrm{T}}+A_{\mathrm{Sis}}$. The intrinsic losses of the tank are given by $A_{\mathrm{T}}=2 \pi W_{\mathrm{T}} / Q_{0}$, where $Q_{0}$ is the intrinsic quality factor of the tank circuit. To estimate the average work done by the tank on the qubit per period, $A_{\text {Sis, }}$, we consider the optimal situation when the oscillator brings the qubit into resonance at the turning point of its trajectory (Fig. 1). We assume further that the state of the qubit is instantaneously 'thermalized', with equal probabilities to remain in the ground state or to get excited. In the latter case, after leaving the area of resonance, the qubit can relax with rate $\Gamma_{\mathrm{R}}$. The probability of the qubit relaxing during one period $2 \pi / \omega_{\mathrm{T}}$ within an interval $\mathrm{d} t$ is given by $\mathrm{d} P=\exp \left(-\Gamma_{\mathrm{R}} t\right) \Gamma_{\mathrm{R}} \mathrm{d} t$. With $I_{\text {r.f. }}(t)=-I_{\mathrm{T}} \cos \left(\omega_{\mathrm{T}} t\right)$ and $\mathrm{d} A_{\mathrm{Sis}}=M I_{\mathrm{p}} \mathrm{d} I_{\text {r.f. }}$, we obtain the Sisyphus work carried out by the oscillator in this event to be $2 M I_{\mathrm{p}} I_{\mathrm{T}}\left(1-\cos \left(\omega_{\mathrm{T}} t\right)\right)$, where the factor 2 is due to the work carried out after the relaxation. Thus, we obtain the value of the work averaged over many cycles (taking into account the probability $1 / 2$ of the initial state to be excited)

$$
A_{\mathrm{Sis}}=M I_{\mathrm{p}} I_{\mathrm{T}} \int \mathrm{d} P\left[1-\cos \left(\omega_{\mathrm{T}} t\right)\right]=M I_{\mathrm{p}} I_{\mathrm{T}} f\left(\Gamma_{\mathrm{R}}, \omega_{\mathrm{T}}\right),
$$

where

$$
f\left(\Gamma_{\mathrm{R}}, \omega_{\mathrm{T}}\right) \equiv\left(1-\mathrm{e}^{-2 \pi \Gamma_{\mathrm{R}} / \omega_{\mathrm{T}}}\right) \frac{\omega_{\mathrm{T}}^{2}}{\omega_{\mathrm{T}}^{2}+\Gamma_{\mathrm{R}}^{2}} .
$$


This estimate demonstrates that the optimal situation for damping or amplification is reached when $\Gamma_{\mathrm{R}} \sim \omega_{\mathrm{T}}$. For the effective quality factor, we obtain

$$
\begin{aligned}
Q_{\text {tank }} & =Q_{0}\left(1 \pm \frac{A_{\mathrm{Sis}} Q_{0}}{2 \pi W_{\mathrm{T}}}\right)^{-1} \\
& =Q_{0}\left(1 \pm \frac{M I_{\mathrm{p}} Q_{0} \omega_{\mathrm{T}} f\left(\Gamma_{\mathrm{R}}, \omega_{\mathrm{T}}\right)}{\pi V_{\mathrm{T}}}\right)^{-1},
\end{aligned}
$$

where \pm stands for damping/amplification. Substituting into equation (4), we arrive at

$$
V_{\mathrm{T}}-V_{\mathrm{T} 0}=\mp M \omega_{\mathrm{T}} I_{\mathrm{p}} Q_{0} f\left(\Gamma_{\mathrm{R}}, \omega_{\mathrm{T}}\right) / \pi,
$$

where $V_{\mathrm{T} 0}=Q_{0} \omega_{\mathrm{T}} L_{\mathrm{T}} I_{\mathrm{T}}^{\mathrm{d}}$ is the voltage on the tank circuit far from resonance. Equation (6) provides the estimate for the increase/decrease of the tank voltage for large driving currents $I_{\mathrm{T}}^{\mathrm{d}}$, such that the qubit spends most of the time away from the resonant excitation area. For weaker driving, when the times spent away and within the excitation area are comparable, the damping is weaker.

\section{TRANSFORMATIONS OF THE HAMILTONIAN}

After transformation to the eigenbasis of the qubit, the Hamiltonian (1) becomes

$$
\begin{aligned}
H= & -\frac{1}{2} \Delta E \sigma_{z}-\hbar \Omega_{\mathrm{R} 0} \cos \left(\omega_{\mathrm{d}} t\right)\left(\sin \zeta \sigma_{z}-\cos \zeta \sigma_{x}\right) \\
& +\hbar \omega_{\mathrm{T}} a^{\dagger} a+g\left(\sin \zeta \sigma_{z}-\cos \zeta \sigma_{x}\right)\left(a+a^{\dagger}\right),
\end{aligned}
$$

with $\tan \zeta=\varepsilon / \Delta$ and $\Delta E \equiv \sqrt{\varepsilon^{2}+\Delta^{2}}$. Because of the large difference of the energy scales between the qubit and the oscillator, $\Delta E \gg \hbar \omega_{\mathrm{T}}$, we can drop within the usual rotating-wave approximation, the longitudinal driving term $-\hbar \Omega_{\mathrm{R} 0} \cos \left(\omega_{\mathrm{d}} t\right) \sin \zeta \sigma_{z}$. On the other hand, we retain the transverse coupling term, $-g \cos \zeta \sigma_{x}\left(a+a^{\dagger}\right)$, but transform it by using a Schrieffer-Wolff transformation, $U_{S}=\exp (i S)$, with generator $S=(g / \Delta E) \cos \zeta\left(a+a^{\dagger}\right) \sigma_{y}$, into a second-order longitudinal coupling. The Hamiltonian then reduces to the form (2).

\section{DISSIPATION IN THE SYSTEM}

The dynamics of the system is strongly influenced by dissipation. The qubit's dissipation is well described by the Lindblad form

$$
L_{\mathrm{Q}} \rho=\frac{\Gamma_{\mathrm{R}}}{2}\left(2 \sigma_{-} \rho \sigma_{+}-\rho \sigma_{+} \sigma_{-}-\sigma_{+} \sigma_{-} \rho\right)+\frac{\Gamma_{\varphi}^{*}}{2}\left(\sigma_{z} \rho \sigma_{z}-\rho\right) .
$$

We can neglect excitation processes because the qubit's energy splitting exceeds the temperature. Thus, the standard longitudinal relaxation rate is given by $T_{1}^{-1}=\Gamma_{\mathrm{R}}$. The rates $\Gamma_{\mathrm{R}}$ and $\Gamma_{\varphi}^{*}$ may depend on the working point, that is, on $\zeta$. In the experiments considered here, this point is fixed by the driving frequency by the condition $\hbar \omega_{\mathrm{d}} \approx \Delta E$. As cooling and amplification appear only in the narrow vicinity of this point, and for all other values of the bias the qubit is in the ground state and its response does not depend on the relaxation rates, it is reasonable to use single values for both $\Gamma_{\mathrm{R}}$ and $\Gamma_{\varphi}^{*}$. It should be mentioned that pure dephasing is frequently caused by the $1 / f$ noise ${ }^{26}$, for which the Markovian description used here is not applicable. We expect, however, that the main features are still captured provided $\Gamma_{\varphi}^{*}$ is chosen properly.
In addition, the resonator damping term can be written in the usual form ${ }^{27}$,

$$
\begin{aligned}
L_{\mathrm{R}} \rho= & \frac{\kappa}{2}\left(N_{\mathrm{th}}+1\right)\left(2 a \rho a^{\dagger}-a^{\dagger} a \rho-\rho a^{\dagger} a\right) \\
& +\frac{\kappa}{2} N_{\text {th }}\left(2 a^{\dagger} \rho a-a a^{\dagger} \rho-\rho a a^{\dagger}\right),
\end{aligned}
$$

where $\kappa=\omega_{\mathrm{T}} / Q_{0}$ characterizes the strength of the resonator damping and $N_{\text {th }}=\left[\exp \left(\hbar \omega_{\mathrm{T}} / k_{\mathrm{B}} T_{0}\right)-1\right]^{-1}$ is the thermal average number of photons in the resonator.

Received 21 December 2007; accepted 6 June 2008; published 6 July 2008.

\section{References}

1. Nakamura, Y., Pashkin, Y. A. \& Tsai, J. S. Coherent control of macroscopic quantum states in a single-cooper-pair box. Nature 398, 786-788 (1999).

2. Vion, D. et al. Manipulating the quantum state of an electrical circuit. Science 296, 886-889 (2002).

3. Chiorescu, I., Nakamura, Y., Harmans, C. \& Mooii, J. Coherent quantum dynamics of a superconducting flux qubit. Science 299, 1869-1871 (2003).

4. Wallraff, A. et al. Strong coupling of a single photon to a superconducting qubit using circuit quantum electrodynamics. Nature 431, 162-167 (2004).

5. Chiorescu, I. et al. Coherent dynamics of a flux qubit coupled to a harmonic oscillator. Nature 431, 159-162 (2004).

6. Il'ichev, E. et al. Continuous monitoring of Rabi oscillations in a Josephson flux qubit. Phys. Rev. Lett. 91, 097906 (2003)

7. Blais, A., Huang, R., Wallraff, A., Girvin, S. M. \& Schoelkopf, R. J. Cavity quantum electrodynamics for superconducting electrical circuits: An architecture for quantum computation. Phys. Rev. A 69, 062320 (2004).

8. Martin, I., Shnirman, A., Tian, L. \& Zoller, P. Ground state cooling of mechanical resonators. Phys. Rev. B 69, 125339 (2004).

9. Niskanen, A. O., Nakamura, Y. \& Pekola, J. P. Information entropic superconducting microcooler. Phys. Rev. B 76, 174523 (2007).

10. Hauss, J., Fedorov, A., Hutter, C., Shnirman, A. \& Schön, G. Single-qubit lasing and cooling at the Rabi frequency. Phys. Rev. Lett. 100, 037003 (2008).

11. Rodrigues, D. A., Imbers, J. \& Armour, A. D. Quantum dynamics of a resonator driven by a superconducting single-electron transistor: A solid-state analogue of the micromaser. Phys. Rev. Lett. 98, 067204 (2007).

12. Astafiev, O. et al. Single artificial-atom laser. Nature 449, 588-590 (2007).

13. Wineland, D. J., Dalibard, J. \& Cohen-Tannouji, C. Sisyphus cooling a bound atom. J. Opt. Soc. B9, 32-42 (1992).

14. Mooij, J. E. et al. Josephson persistent-current qubit. Science 285, 1036-1039 (1999).

15. Quan, H. T., Liu, Y. X., Sun, C. P. \& Nori, F. Quantum thermodynamic cycles and quantum heat engines. Phys. Rev. E 76, 031105 (2007).

16. Greenberg, Y. S. et al. Method for direct observation of coherent quantum oscillations in a superconducting phase qubit. Phys. Rev. B 66, 224511 (2002).

17. Grajcar, M., Ashhab, S., Johansson, J. \& Nori, F. Lower limit on the achievable temperature in resonator-based sideband cooling. Preprint at $<$ http://arxiv.org/abs/0709.3775 > (2007).

18. Irish, E. K. \& Schwab, K. Quantum measurement of a coupled nanomechanical resonator-Cooper-pair box system. Phys. Rev. B 68, 155311 (2003).

19. Schuster, D. I. et al. Resolving photon number states in a superconducting circuit. Nature $\mathbf{4 4 5}$ 515-518 (2007)

20. Gambetta, J. et al. Qubit-photon interactions in a cavity: Measurement-induced dephasing and number splitting. Phys. Rev. A 74, 042318 (2006).

21. LaHaye, M. D., Buu, O., Camarota, B. \& Schwab, K. C. Approaching the quantum limit of a nanomechanical resonator. Science 304, 74-77 (2004).

22. Blanter, Y. M., Usmani, O. \& Nazarov, Y. V. Single-electron tunnelling with strong mechanical feedback. Phys. Rev. Lett. 93, 136802 (2004).

23. Blencowe, M. P., Imbers, J. \& Armour, A. D. Dynamics of a nanomechanical resonator coupled to a superconducting single-electron transistor. New J. Phys. 7, 236 (2005).

24. Naik, A. et al. Cooling a nanomechanical resonator with quantum back-action. Nature $\mathbf{4 4 3}$, 193-196 (2006).

25. Bennett, S. D. \& Clerk, A. A. Laser-like instabilities in quantum nano-electromechanical systems. Phys. Rev. B 74, 201301 (2006)

26. Ithier, G. et al. Decoherence in a superconducting quantum bit circuit. Phys. Rev. B 72 , 134519 (2005).

27. Gardiner, C. W. \& Zoller, P. Quantum noise 3rd edn (Springer, Berlin, 2004).

\section{Acknowledgements}

M.G. was supported by Grants VEGA 1/0096/08 and APVV-0432-07. We further acknowledge the financial support from the EU (RSFQubit and EuroSQIP).

\section{Author information}

Reprints and permission information is available online at http://npg.nature.com/reprintsandpermissions. Correspondence and requests for materials should be addressed to M.G. 